\title{
Constraining the Nature of the Galactic Center X- Ray Source Population
}

\section{Citation}

Laycock, S., J. Grindlay, M. van den Berg, P. Zhao, J. Hong, X. Koenig, E. M. Schlegel, and S. E. Persson. 2005. "Constraining the Nature of the Galactic Center X-Ray Source Population." The Astrophysical Journal 634 (1): L53-56. https://doi.org/10.1086/498821.

\section{Permanent link}

http://nrs.harvard.edu/urn-3:HUL.InstRepos:41399919

\section{Terms of Use}

This article was downloaded from Harvard University's DASH repository, and is made available under the terms and conditions applicable to Other Posted Material, as set forth at http:// nrs.harvard.edu/urn-3:HUL.InstRepos:dash.current.terms-of-use\#LAA

\section{Share Your Story}

The Harvard community has made this article openly available.

Please share how this access benefits you. Submit a story.

Accessibility 


\title{
CONSTRAINING THE NATURE OF THE GALACTIC CENTER X-RAY SOURCE POPULATION
}

\author{
S. Laycock, ${ }^{1}$ J. Grindlay, ${ }^{1}$ M. van den Berg, ${ }^{1}$ P. Zhao, ${ }^{1}$ J. Hong, ${ }^{1}$ X. Koenig, ${ }^{1}$ E. M. Schlegel, ${ }^{1}$ And S. E. Persson ${ }^{2}$ \\ Received 2005 June 11; accepted 2005 October 13; published 2005 November 3
}

\begin{abstract}
We searched for infrared counterparts to the cluster of X-ray point sources discovered by Chandra in the Galactic center region (GCR). While the sources could be white dwarfs, neutron stars, or black holes accreting from stellar companions, their X-ray properties are consistent with magnetic cataclysmic variables (CVs), or highmass X-ray binaries (HMXBs) at low accretion rates. A direct way to decide between these possibilities, and hence between alternative formation scenarios, is to measure or constrain the luminosity distribution of the companions. Using infrared $(J, H, K, \mathrm{Br} \gamma)$ imaging, we searched for counterparts corresponding to typical HMXB secondaries: spectral type B0 V with $K<15$ at the GCR. We found no significant excess of bright stars in Chandra error circles, indicating that HMXBs are not the dominant X-ray source population and that they account for fewer than $10 \%$ of the hardest X-ray sources.
\end{abstract}

Subject headings: Galaxy: center $-\mathrm{X}$-rays: binaries

Online material: color figure

\section{INTRODUCTION}

With the high spatial resolution of Chandra and groundbased infrared (IR) instrumentation, the Galactic center, heavily obscured by dust, is now accessible to a wide range of astrophysical investigations. A series of Chandra observations of the GCR $\left(\sim 10^{\prime}\right.$ region around Sgr A*), totaling $670 \mathrm{ks}$, have recently been analyzed by Muno et al. (2003, hereafter M03), revealing 2357 point sources. The $2-8 \mathrm{keV} \mathrm{X}$-ray luminosity $\left(10^{31}-10^{33} \mathrm{ergs} \mathrm{s}^{-1}\right)$ and spectral index $(\Gamma<1)$ of the majority of the sources are inconsistent with normal stars, active binaries, or young stellar objects, none of which are normally persistently luminous enough at $>2.5 \mathrm{keV}$ to produce the observed fluxes in the presence of the high absorption column $\left(N_{\mathrm{H}} \sim 10^{23} \mathrm{~cm}^{-2}\right)$. The only sources expected to be detected given the absorption are compact objects accreting from binary companions. The luminosity range of the GCR sources covers magnetic CVs and non-Roche lobe filling HMXBs accreting in a low mass transfer regime, e.g., X Per and A0535+26 (Negueruela et al. 2000). Quiescent low-mass X-ray binaries (LMXBs) with neutron star (NS) or black hole (BH) primaries are possible contributors but are generally too faint $(\mathrm{BHs})$ or too soft (NSs). HMXBs are young objects (a few megayears), while CVs are slow to form and long-lived. The two possibilities point to different evolutionary phases of the GCR.

If HMXBs comprise a significant fraction of the GCR sources, they must be the products of recent, massive star formation. Frequent starburst events in the GCR are expected on $\sim 20$ Myr scales based on the instability of large gas clouds orbiting within $200 \mathrm{pc}$ of the nucleus (Stark et al. 2004) and the presence of several young clusters of massive stars. The HMXB hypothesis was proposed and discussed in detail by Pfahl et al. (2002).

Grindlay (1985) suggested that the Galactic bulge contains the debris of tidally disrupted globular clusters. Were the GCR sources dominated by magnetic CVs, globular clusters would then contribute because CVs are overproduced per unit mass in globulars relative to the Galactic plane.

\footnotetext{
${ }^{1}$ Harvard-Smithsonian Center for Astrophysics, 60 Garden Street, Cambridge, MA 02138.

${ }^{2}$ Observatories of the Carnegie Institution of Washington, 813 Santa Barbara Street, Pasadena, CA 91101.
}

Morris (1993) proposed a scenario in which compact stellar remnants form in the Galactic bulge and sink toward the center of the dense mass distribution via gravitational interactions. The presence of a supermassive $\mathrm{BH}$ at the center accelerates the process, leading to a central density cusp. This scenario fits with the Muno et al. (2005, hereafter M05) discovery of a centrally concentrated overabundance of X-ray transients as well as the overall $1 / \theta$ source distribution found by M03.

All three channels may be active now or have been in the past, so by measuring relative numbers of the various endproducts, we hope to piece together the composition and history of the GCR. In this Letter we present preliminary results of our deep IR survey targeted at measuring the HMXB fraction. The occurrence of IR counterparts consistent with highly reddened massive stars and giants will constrain the relative abundance of HMXBs and CVs. This work is part of our Chandra Multi-wavelength Plane Survey (ChaMPlane; Grindlay et al. 2005), which is an effort to map the space density of all types of low-luminosity accretion sources in the Galaxy.

\section{OBSERVATIONS}

The GCR is heavily obscured with average $A_{V} \sim 25$, so counterparts can only be detected with IR imaging. The stellar density is also very high so that reaching a sufficient limiting magnitude to make meaningful constraints on population models demands excellent image quality. In 2004June, we observed the inner $10^{\prime} \times 10^{\prime}$ of the GCR with the PANIC (Persson's Auxiliary Nasmyth Infrared Camera) imager on the $6.5 \mathrm{~m}$ Magellan (Baade) telescope at Las Campanas, Chile. PANIC has a $2^{\prime} \times 2^{\prime}$ field of view with $0^{\prime \prime} 125$ pixels. Under good seeing conditions (median FWHM $\sim 0$ ".5), we obtained a mosaic of 25 pointings in $J, H, K_{s}$, and $\mathrm{Br} \gamma$ filters using a five-point dither pattern with three exposures at each position. Sky emission was removed using off-source exposures, and the images were reduced and stacked in IRAF. Photometry was performed on the stacked images using SExtractor (Bertin \& Arnouts 1996). Astrometric and photometric calibrations were performed using the Two Micron All Sky Survey (2MASS) point-source catalog.

We used a version of the X-ray point-source catalog of M03, kindly provided by M. Muno, listing J2000 coordinates with the net count rate, flux, and source significance determined in 


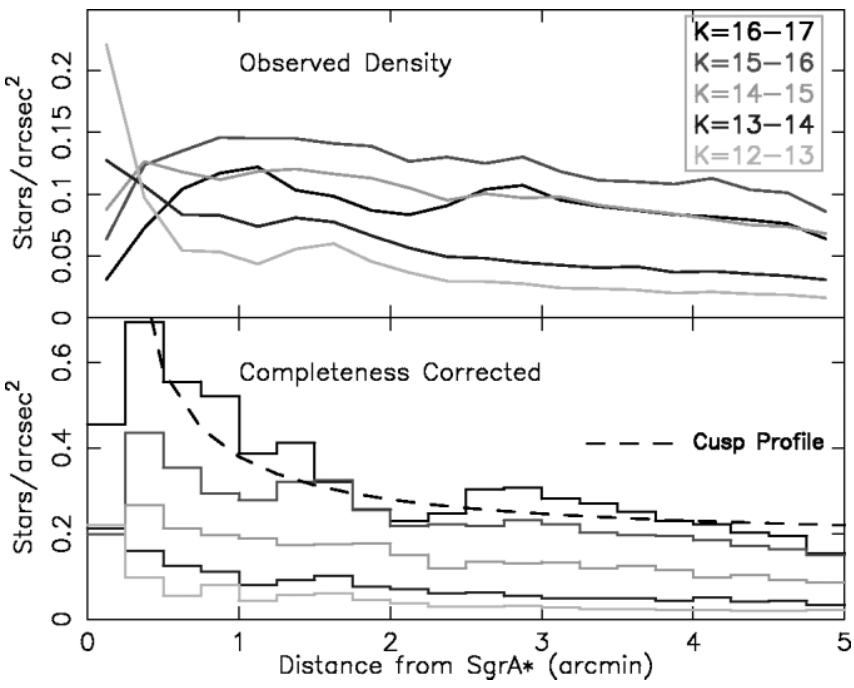

FIG. 1.-Star density curves for PANIC $K$-band photometry, in five magnitude ranges as indicated in the top panel inset. Top panel: Observed number density shown as curves. Bottom panel: Completeness-corrected upper limits shown as histograms. A radial profile of the form $\mathrm{A}+\mathrm{B} \times \theta^{-1}$ is also shown. [See the electronic edition of the Journal for a color version of this figure.]

three energy bands: S1 (0.3-1.2 keV), S2 (1.2-2.5 keV), and $\mathrm{H}(2.5-8 \mathrm{keV})$. The intermediate $\mathrm{S} 2$ band provides some additional information, but for this Letter we restrict ourselves to discussion of GCR (H only) and foreground (S1) sources.

\section{EXPERIMENTAL DESIGN}

We set out to identify all potential IR counterparts to the M03 X-ray sources and apply statistical tests to measure their numbers relative to random positional coincidence. Our scientific goals are to set stringent upper limits on the number of counterparts and examine their photometric properties.

The availability of X-ray spectral information and multiband IR photometry permits us to cleanly separate sources situated in the GCR from those in the foreground. The GCR sources are characterized by X-ray spectra completely cut off at low energies due to absorbing gas and dust. Of the sources in the M03 catalog, $80 \%$ are detected at $>3 \sigma$ in the $\mathrm{H}$ band only, while any source detected in the S1 band must lie in the foreground. The spatial distributions of X-ray point sources and $K$-band stars follow similar radial profiles about the GCR. M03 demonstrated a surface density with $1 / \theta$ dependence with an angular offset $\theta$ from Sgr A*. Similar results have been reported for stars from IR observations (e.g., Catchpole et al. 1990).

There are 100 known Galactic HMXBs, of which $70 \%$ have Be star companions whose spectral types lie in the narrow range O9-B2 and peak at B0 V (Negueruela 1998). We adopt the luminosity and color of a B0 V to represent "typical" HMXB values $\left(M_{V}=-4, V-K=-0.83\right.$; $\left.\operatorname{Cox} 2000\right)$. Note that many non-Be HMXBs are more luminous because they have supergiant companions. For the $\mathrm{CV}$ case, we adopt a K0 V dwarf $\left(M_{V}=5.9, V-K=1.96\right)$ at the brighter end of the normal range (Patterson 1998). Adopting $A_{V}=25$ and $D=$ $8 \mathrm{kpc}$ for the GCR, our target HMXB should have an apparent magnitude $K=14.4$ while the assumed $\mathrm{CV}$ would have $K=21$. We note the intermediate polar GK Per with bright subgiant counterpart would have $K \sim 19$.

\subsection{Photometric Completeness Curve}

Due to severe crowding in the GCR, we infer the star density from our observations by correcting for incompleteness, i.e.,
TABLE 1

Peak-up Test on the Full $10^{\prime} \times 10^{\prime}$ Field

\begin{tabular}{|c|c|c|c|c|c|c|c|c|}
\hline$K_{1}-K_{2}$ & $N_{m}$ & $N_{\text {ran }}$ & $\sigma$ & $N_{\text {exc }}$ & Sig. & $C$ & $U_{90}$ & $U_{c}$ \\
\hline \multicolumn{9}{|c|}{ Hard Sources (1453) } \\
\hline$>12$ & 399 & 353 & 15.8 & 46 & 2.9 & $\ldots$ & $87 *$ & $\ldots$ \\
\hline$>17$ & 86 & 77.9 & 8.1 & 8.1 & 1.0 & & $20 *$ & \\
\hline $16-17$ & 72 & 57.7 & 7.2 & 14.3 & 2.0 & 0.3 & 54 & 10.7 \\
\hline $15-16$ & 84 & 83.0 & 9.3 & 1.0 & 0.1 & 0.5 & 27 & 7.3 \\
\hline $14-15$ & 83 & 67.5 & 8.3 & 15.5 & 1.9 & 0.7 & 37 & 5.4 \\
\hline $13-14$ & 37 & 35.7 & 6.1 & 1.3 & 0.2 & 0.8 & 10 & 3.0 \\
\hline $12-13$ & 30 & 22.6 & 4.5 & 7. & 1.6 & 0.9 & 13 & 2.3 \\
\hline $11-12$ & 11 & 8.6 & 2.4 & 2.4 & 1.0 & 1.0 & 11 & 1.4 \\
\hline $10-11$ & 6 & 5.3 & 1.9 & 0.7 & 0.4 & 1.0 & 6 & 0.6 \\
\hline $9-10$ & 2 & 2.2 & 1.3 & -0.2 & -0.2 & 1.0 & 2 & 0.2 \\
\hline \multicolumn{9}{|c|}{ Soft Sources (105) } \\
\hline$>12$ & 67 & 28.6 & 4.7 & 38.4 & 8.2 & & $59 *$ & \\
\hline $15-16$ & 8 & 6.2 & 2.6 & 1.8 & 0.7 & 0.5 & 10 & 92 \\
\hline $14-15$ & 21 & 5.3 & 2.3 & 15.7 & 6.8 & 0.7 & 27 & 84 \\
\hline $13-14$ & 10 & 2.6 & 1.4 & 7.4 & 5.3 & 0.8 & 11 & 59 \\
\hline $12-13$ & 13 & 1.5 & 1.3 & 11.5 & 8.8 & 0.9 & 14 & 49 \\
\hline $11-12$ & 21 & 0.4 & 0.7 & 20.6 & 29.4 & 1.0 & 21 & 35 \\
\hline $10-11$ & 12 & 0.2 & 0.5 & 11.8 & 23.6 & 1.0 & 12 & 15 \\
\hline $9-10 \ldots$ & 4 & 0.0 & 0.1 & 4.0 & 40 & 1.0 & 4 & 4 \\
\hline
\end{tabular}

Notes. - In the $\sim 10^{\prime} \times 10^{\prime}$ PANIC survey area, we consider $184 \times 10^{3} \mathrm{~K}$ band stars, 1453 hard X-ray sources, and 105 soft X-ray sources. The 2MASS point-source catalog was used for $K<12 . N_{m}$ is the number of X-ray sources with one or more matches. $N_{\text {ran }}$ is the number of random matches, and $\sigma$ is the uncertainty in the number of random matches. $N_{\mathrm{exc}}$ is the number of "excess" matches above the random level. Sig. is the significance of excess. $C$ is the completeness fraction for $K$-band stars. $U_{90}$ is the completenesscorrected upper limit on the number of counterparts at $90 \%$ significance. $U_{c}$ is the cumulative $U_{90}$ brightward of $K_{2}$ expressed as a percentage of the number of X-ray sources. The asterisk indicates that no completeness correction was applied for unbounded magnitude ranges.

the loss of faint stars due to their brighter neighbors. Completeness curves for our PANIC photometry as functions of magnitude and distance from Sgr A* were determined by simulation. For each of the $25 \mathrm{~K}$-band-stacked images, a random sample of stars was added using IRAF mkstar with a Gaussian point-spread function matched to the seeing. The photometry was repeated, and the artificial stars identified and compared to their input magnitudes. A master catalog of input and recovered stars was then assembled, from which completeness curves could be extracted for any region of the $10^{\prime} \times 10^{\prime} \mathrm{mo}-$ saic. The $50 \%$ completeness limit (the fraction of stars recovered within \pm 0.5 mag of their input magnitude) within the inner $1^{\prime}$ radius occurs at $K=14.5$, and over the rest of the mosaic at $K=15.4$. Curves for star density are presented in Figure 1, and completeness values for the full field are reported in the final column of Table 1.

\subsection{Peak-up Test}

The IR and X-ray catalogs are repeatedly matched over a large grid of positional offsets using integer multiples of the PANIC pixel size of $00^{\prime \prime} 125$. At each offset position, the number of X-ray sources with one or more stars lying in their error circle is recorded in a series of magnitude bins. We used $95 \%$ confidence error circles determined from the formula of Hong et al. (2005) that is dependent on net counts and distance from the Chandra aim point. For the GCR sources, error radii are $0.3-1$. The presence of real counterparts is signaled by a peak at the offset coordinates corresponding to perfect alignment of the two catalogs, providing a boresight. To confirm that X-ray and IR catalogs are on the same astrometric frame, we centroided the soft-source peak, finding a residual offset of $\Delta$ R.A. $=00^{\prime \prime} 011(1), \Delta$ decl. $=00^{\prime \prime 092(1) ~ w i t h ~ F W H M ~}=0.55$. 
The number of random matches $\left(N_{\text {ran }}\right)$ is calculated as the mean of all trials at shifts greater than $2^{\prime \prime}$, i.e., far away from correct alignment, and the standard deviation $(\sigma)$ of these values is used to characterize the uncertainty. We then take the (peak) number of matches $\left(N_{m}\right)$ and subtract the random level to obtain the number of excess-above-random matches $\left(N_{\text {exc }}\right)$. We claim detection of a population of counterparts above that predicted to occur by chance if $N_{\mathrm{exc}}$ exceeds $3 \sigma$. The upper limit on the total number of true counterparts was determined by a Monte Carlo simulation. At each iteration, the X-ray catalog was offset by randomly generated shifts in $X$ and $Y$ such that the absolute shift was between $2^{\prime \prime}$ and $5^{\prime \prime}$. A randomly selected sample of $N_{\text {true }}$ X-ray sources were flagged as "true counterparts," and the catalogs matched as previously described. Since all of the Xray/IR matches were by definition random (due to the offsets), we were able to count the total number of matches $N_{\text {sim }}$ in each magnitude bin, correcting for duplicates (sources flagged as simulated matches that also happened to have a random IR counterpart) such that $N_{\text {sim }}=N_{\text {ran }}+N_{\text {true }}-N_{\text {ran+true }}$. Guided by the values of $\sigma$ from the peak-up test, we ran simulations with $N_{\text {true }}=5-40$, with 100 iterations at each value. For each value of $N_{\text {true }}$, we recorded the largest value of $N_{\text {sim }}$ reached in $90 \%$ of the trials, and we fitted the results with a straight line relationship. Thus, the upper limit on the number of true matches corresponds to the value of $N_{\text {true }}$ when $N_{\text {sim }}=N_{m}$; these values are given in Table 1 as $U_{90}$.

\section{CONSTRAINTS ON IR/X-RAY COUNTERPARTS}

We performed the peak-up test both on the entire field and, independently, on a series of concentric annular regions centered on Sgr A*. This allows us to limit the effects of crowding in the IR and to follow the cusp distribution of hard X-ray sources.

For the full field we found no significant excess in the number of IR matches for hard X-ray sources, irrespective of magnitude, while for soft sources we found a highly significant $(\sim 30 \sigma)$ counterpart population dominated by bright $(K \sim 11)$ stars. This result is illustrated in Figure 2 and, in more detail, in Table 1 . There is a marginal signal $(2.9 \sigma)$ for hard sources versus the entire PANIC catalog, which could not be associated with any specific magnitude range.

We therefore conclude that the GCR counterpart population is dominated by stars fainter than our confusion limit, although some fraction of the hard matches are undoubtedly real. The $90 \%$ confidence upper limit for the full catalog indicates that there are up to 87 hard counterparts, although we cannot at this stage identify them from among the 399 total matches. By placing completeness-corrected upper limits on the number of counterparts as a function of $K$ magnitude, we can constrain the proportion of HMXBs and hence place a lower limit on the population of CVs and LMXBs. The final column of Table 1 gives the cumulative percentage obtained by adding the upper limits for each magnitude range and dividing by the number of $\mathrm{X}$-ray sources. We find that a maximum of $5.4 \%$ (at $90 \%$ confidence) of the sources could have counterparts brighter than $K=15$, given the average GCR extinction. The presence of a significant HMXB population would appear between $K=14$ and 16, and the fact that we do detect soft-source counterparts for $K=14-15$ (at $6.8 \sigma$ ) demonstrates that we are not merely being defeated by crowding in this range.

For annular bins $0-1^{\prime}, 1^{\prime}-2^{\prime}, 2^{\prime}-3^{\prime}, 3^{\prime}-4^{\prime}$, and $4^{\prime}-5^{\prime}$, our results constrain the $\mathrm{HMXB}$ population as a function of distance from $\operatorname{Sgr} \mathrm{A}^{*}$. The effect of the nuclear cluster is apparent in the inner $1^{\prime}$, where a large number of massive stars raise the

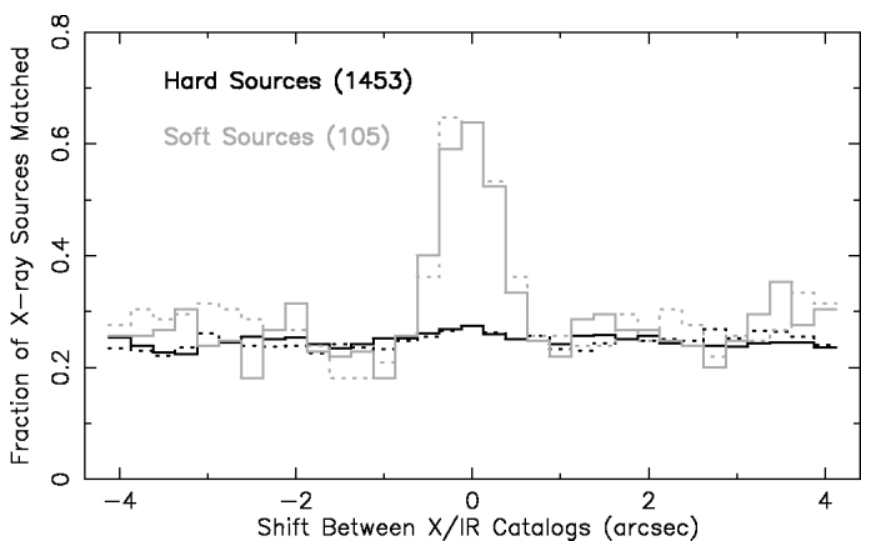

FIG. 2.-Peak-up test for hard and soft X-ray sources, using PANIC $K$-band photometry. The solid and dotted lines show projections along the right ascension and declination axes of the number of X-ray sources, with one or more stars inside the $95 \%$ error circle. A prominent peak is seen at zero offset only for the soft sources. The random match level and its fluctuations are apparent beyond the $1^{\prime \prime}$ offset.

upper limits such that $K<15 \mathrm{HMXB}$ can potentially account for $9 \%$ of the 110 hard X-ray sources present (at $90 \%$ confidence). The limit is 5\%-8\% for the remaining annuli. Overall, the annular results are less constraining than the full-field results due to the smaller sample sizes involved. A single annular bin $\left(2^{\prime}-3^{\prime}\right)$ produced a peak at $3.8 \sigma$ significance, possibly corresponding to a drop in the confusion limit sufficient to allow counterparts to be isolated. Such an effect could be due to seeing, anomalous extinction, or an unrelated star cluster.

We can account for $84 \%$ of the 105 soft sources down to $K<15$, and essentially all to $K<16$ where crowding dominates. The majority of soft sources will be dMe and other foreground coronal stars. For example, at spectral type M0 V, our $50 \%$ completeness limit of $K \sim 15.4$ implies a distance of just $1 \mathrm{kpc}$; indeed, $86 \%$ of the $\mathrm{S} 1$ sources have counterparts from our optical ChaMPlane survey (Zhao et al. 2003).

\section{PHOTOMETRY OF POTENTIAL COUNTERPARTS}

Color-magnitude diagrams (CMDs) were constructed to compare the $H-K$ versus $K$ distribution for stars lying in Xray error circles with that of the field population. Extinction due to dust lying between us and the Galactic center makes the GCR stars appear systematically redder than foreground stars, largely overcoming any degeneracy between intrinsic brightness and distance. In Figure 3 we see that the soft counterparts are mainly unreddened stars while the hard counterparts cluster around $H-K \sim 1.6$ with a large scatter. Intrinsic $H-K$ colors do not exceed $\sim+0.3$ for any stars (Cox 2000). Soft-source counterparts are also brighter than average field stars; the Kolmogorov-Smirnov probability that they are the same is $2.6 \times 10^{-9}$. Consistent with the X-ray selection and peak-up test, the soft sources are shown to lie in the foreground, while the hard sources are dominated by random matches to stars in the GCR.

Matches were found for three of the eight periodic X-ray sources seen by Muno et al. (2004, hereafter M04), seven in the PANIC field and five observed in $K_{s}$ (see Fig. 3). One of these (CXOGCR J174534.5-290201) is heavily reddened, while the others have slightly lower $H-K$ values than the main concentration of stars in the CMD. The colors of the candidate stars do not agree with the predicted extinction from X-ray spectral fits of M04. The X-ray-derived $N_{\mathrm{H}}$ of $\gtrsim 10^{23} \mathrm{~cm}^{-2}$ requires the stars to be extremely bright, in order to explain the observed $K$ 


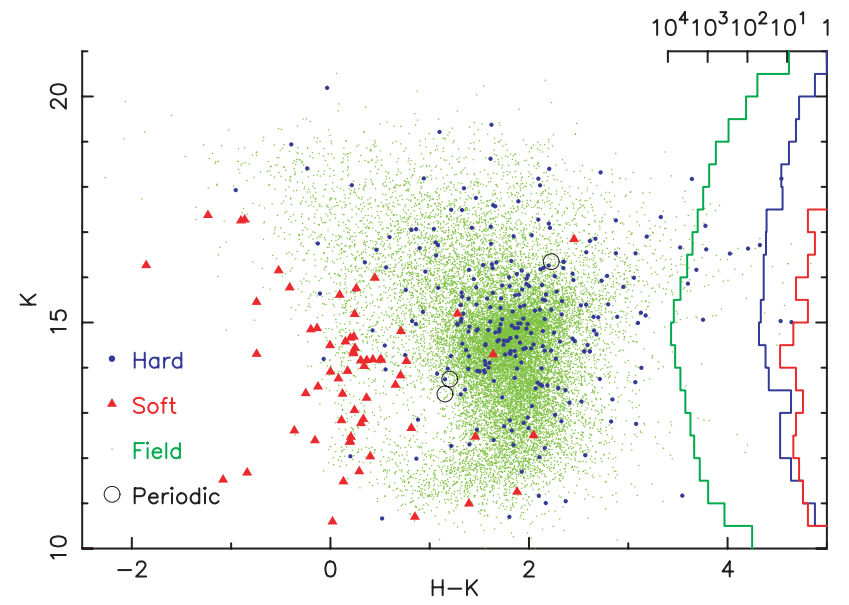

FIG. 3.-CMD comparing the IR counterparts of hard and soft X-ray sources with the field population. The total number in each class is given as a histogram to the right. (Saturation begins at $K \lesssim 11.5$.)

magnitudes, and predicts $H-K$ colors 2 mag redder than what is observed. These observations may be reconciled if most of the X-ray-derived $N_{\mathrm{H}}$ arises in the binary, in an accretion column or disk obscuring the X-ray source. A column of a few times $10^{22} \mathrm{~cm}^{-2}$ is typical for HMXB pulsars but is insufficient to make up the difference. It is also possible that these matches are random and that the real counterparts are much fainter. No match was found for the seven bright transients detected by M05.

A preliminary search for $\mathrm{Br} \gamma$ emission objects among X-ray IR matches yielded 11 candidates with $\mathrm{S} / \mathrm{N}>5$, of which three are foreground objects. $\mathrm{Br} \gamma-K$ is severely restricted by the small amplitude of the expected signal $[\sim-0.1 \mathrm{mag}$ for $\mathrm{EW}(\mathrm{Br} \gamma) \sim 10-20 \AA)$ and the effects of crowding.

\section{CONCLUSIONS}

The highly absorbed GCR X-ray sources appear to be dominated by a population of accreting binaries in which the mass donor is less massive than in a typical HMXB. This conclusion follows from the lack of a significant excess of stars brighter than $K \lesssim 15$ in the error circles of hard X-ray sources, which is the apparent magnitude of a B0 V star at the distance and mean $A_{V}$ of the GCR. By applying our peak-up test as a function of magnitude and distance from $\mathrm{Sgr} \mathrm{A}^{*}$, and correcting for completeness, we set $90 \%$ confidence upper limits on the number of hard X-ray sources that can have counterparts in a series of ranges between $K=9$ and 16. After accumulating these limits for all counterparts brighter than $K=15$, such stars can account for at most 79 out of 1453 hard X-ray sources, or $5.4 \%$. Pushing 1 mag fainter to include Be HMXBs as late as B2, we find an upper limit of 103 , or $7.1 \%$. Within $1^{\prime}$ of Sgr $\mathrm{A}^{*}$, these upper limits become $9 \%$ and $15 \%$ due to the high density of very luminous stars in the nuclear cluster. We note that large variations in $A_{V}$ and/or star clustering could modify these numbers.

Our constraints imply that non-HMXBs account for more than $90 \%$ of the M03 X-ray sources (at least beyond $1^{\prime}$ ) favoring the leading alternative hypothesis of magnetic CVs. High angular resolution spectroscopy will be essential to identify the true counterparts that likely exhibit $\mathrm{B} \gamma$ or $\mathrm{He}$ emission lines indicative of an accretion disk.

This work was funded in part by Chandra grant AR4-5003A and NSF grant AST-0098683. We thank the referee M. Muno for his insightful suggestions.

\section{REFERENCES}

Bertin, E., \& Arnouts, S. 1996, A\&AS, 117, 393

Catchpole, R. M., Whitelock, P. A., \& Glass, I. S., 1990, MNRAS, 247, 479

Cox, A. N., ed. 2000, Allen's Astrophysical Quantities (4th ed.; New York: Springer)

Grindlay, J. E. 1985, in IAU Symp. 113, Dynamics of Star Clusters, ed. J. Goodman \& P. Hut. (Dordrecht: Kluwer), 43

Grindlay, J. E., et al. 2005, ApJ, in press

Hong, J., et al. 2005, ApJ, in press

Muno, M. P., Pfahl, E., Baganoff, F. K., Brandt, W. N., Ghez, A., Lu, J., \& Morris, M. R. 2005, ApJ, 622, L113 (M05)

Muno, M. P., et al. 2003, ApJ, 589, 225 (M03)
Muno, M. P., et al. 2004, ApJ, 613, 1179 (M04)

Morris, M. 1993, ApJ, 408, 496

Negueruela, I. 1998, A\&A, 338, 505

Negueruela, I., Reig, P., Finger, M. H., \& Roche, P. 2000, A\&A, 356, 1003

Patterson, J. 1998, PASP, 110, 1132

Pfahl, E., Rappaport, S., \& Podsiadlowski, P. 2002, ApJ, 571, L37

Stark, A. A., Martin, C. L., Walsh, W. M., Xiao, K., Lane, A. P., \& Walker, C. K. 2004, ApJ, 614, L41

Zhao P., Grindlay, J. E., Hong, J., Laycock, S., Baganoff, F. K., Muno, M. P., Garmire, G. P., \& Morris, M. 2003, BAAS, 7, 37.04 